\title{
BMJ Open Deprescribing fall-risk increasing drugs (FRIDs) for the prevention of falls and fall-related complications: a systematic review and meta-analysis
}

\author{
Justin Lee (1) , ${ }^{1,2,3}$ Ahmed Negm, ${ }^{3,4}$ Ryan Peters, ${ }^{5}$ Eric K C Wong, ${ }^{6}$ \\ Anne Holbrook (1D) ${ }^{2,7}$
}

To cite: Lee J, Negm A, Peters R, et al. Deprescribing fall-risk increasing drugs (FRIDs) for the prevention of falls and fall-related complications: a systematic review and meta-analysis. BMJ Open 2021;11:e035978. doi:10.1136/ bmjopen-2019-035978

- Prepublication history and additional materials for this paper are available online. To view these files, please visit the journal online (http://dx.doi. org/10.1136/bmjopen-2019035978).

Received 28 November 2019 Revised 20 November 2020 Accepted 26 November 2020

Check for updates

(C) Author(s) (or their employer(s)) 2021. Re-use permitted under CC BY-NC. No commercial re-use. See rights and permissions. Published by BMJ.

For numbered affiliations see end of article.

Correspondence to

Dr Justin Lee;

justin.lee@medportal.ca

\section{ABSTRACT}

Objectives Prevention of falls and fall-related injuries is a priority due to the substantial health and financial burden of falls on patients and healthcare systems. Deprescribing medications known as 'fall-risk increasing drugs' (FRIDs) is a common strategy to prevent falls. We conducted a systematic review to determine its efficacy for the prevention of falls and fall-related complications. Design Systematic review and meta-analysis. Data sources MEDLINE, EMBASE, Cochrane Central Register of Controlled Trials, CINAHL and grey literature from inception to 1 August 2020.

Eligibility criteria for selecting studies Randomised controlled trials of FRID withdrawal compared with usual care evaluating the rate of falls, incidence of falls, fall-related injuries, fall-related fractures, fallrelated hospitalisations or adverse effects related to the intervention in adults aged $\geq 65$ years.

Data extraction and synthesis Two reviewers independently performed citation screening, data abstraction, risk of bias assessment and certainty of evidence grading. Random-effects models were used for meta-analyses.

Results Five trials involving 1305 participants met eligibility criteria. Deprescribing FRIDs did not change the rate of falls (rate ratio (RaR) $0.98,95 \% \mathrm{Cl} 0.63$ to 1.51 ), the incidence of falls (risk difference $0.01,95 \% \mathrm{Cl}-0.06$ to 0.09 ; relative risk $1.04,95 \% \mathrm{Cl} 0.86$ to 1.26 ) or rate of fall-related injuries ( $\mathrm{RaR} 0.89,95 \% \mathrm{Cl} 0.57$ to 1.39 ) over a follow-up period of 6-12 months. No trials evaluated the impact of deprescribing FRIDs on fall-related fractures or hospitalisations.

Conclusion There is a paucity of robust high-quality evidence to support or refute that a FRID deprescribing strategy alone is effective at preventing falls or fall-related injury in older adults. Although there may be other reasons to deprescribe FRIDs, our systematic review found that it may result in little to no difference in the rate or risk of falls as a sole falls reduction strategy.

PROSPERO registration number CRD42016040203.

\section{INTRODUCTION}

Falls and fall-related injuries are significant public health concerns. Every year, one in three older adults aged $\geq 65$ years falls and
Strengths and limitations of this study

- This study's results are based on a systematic review and meta-analysis of randomised controlled trials.

- We employed rigorous analytical methods and interpretational approaches including duplicate assessment, subgroup credibility criteria and optimal information size considerations.

- We assessed the certainty in evidence (ie, quality of evidence) using the Grading of Recommendations, Assessment, Development and Evaluation Framework

- Additional studies are needed to reach the optimal information size to reduce uncertainty about this intervention and establish its relative importance in the range of possible falls prevention interventions.

$10 \%$ of these falls cause serious injury or hospitalisation. ${ }^{1}$ Falls are estimated to annually cost $\$ 50$ billion in the USA, $\$ 2$ billion in Canada and £2.3 billion in the UK. ${ }^{2-4}$ All jurisdictional levels are making significant investments to implement falls prevention quality improvement initiatives. These include Public Health England's National Falls Prevention Coordinating Group, the Centers for Disease Control and Prevention's Stopping Elderly Accidents, Deaths and Injuries Initiative, and Health Canada's Canadian Patient Safety Institute 'Reducing Falls and Injuries from Falls' initiative. National accreditation bodies such as the US Joint Commission and Accreditation Canada also mandate specific falls prevention activities of healthcare organisations through their required organisational practices and standards.

Since the majority of falls result from multiple factors (eg, poor strength and balance, visual and cognitive impairment), current practice guidelines and accreditation standards focus on multifactorial assessment 
and intervention strategies. ${ }^{5}$ These strategies involve the combination of two or more interventions (eg, exercise, home or environmental modification, vision assessment, education, medication management, vitamin D supplementation). However, the 2018 US Preventive Services Task Force evidence report recommends that multifactorial interventions only be offered to select patients because the overall net benefit is small. ${ }^{6}$ In fact, there is ongoing debate on the relative merits of focusing on single versus multifactorial interventions, and many clinicians and institutions focus on single interventions due to limited resources. ${ }^{7}$

As an individual intervention, only exercise has robust evidence demonstrating reductions in the incidence of fallers and rate of injurious falls. ${ }^{68}$ It is unclear if other parts of the multicomponent strategy are effective, how large is their individual treatment effect and which components should be prioritised when resources are limited.

Although there is limited evidence of effectiveness, deprescribing medications known as 'fall-risk increasing drugs' (FRIDs) is common practice and typically included in both multifactorial and single intervention strategies. The justification is based on observational studies that suggest certain medications are associated with increased falls risk as well as some randomised controlled trials (RCTs) that have shown that medication management interventions (including those with a broader focus of reducing polypharmacy and/or potentially inappropriate prescribing) may reduce the risk of falls. ${ }^{9}$ FRIDs include antihypertensives, antiarrhythmics, anticholinergics, antihistamines, sedatives-hypnotics, antipsychotics, antidepressants, opioids and non-steroidal anti-inflammatory drugs. ${ }^{10-15}$ Although the mechanisms are not fully understood, these drugs may influence falls risk by adversely affecting the cardiovascular or central nervous system (eg, orthostatic hypotension, bradycardia, sedation, sleep disturbance, confusion, dizziness).

Key issues affecting the quality of this observational evidence and certainty of a causal relationship include: (1) variable adjustment for confounders, dosage or duration of therapy, (2) medication use confirmed only at baseline (but not throughout follow-up) and (3) potential prescribing bias associated with specific medication classes. Most meta-analyses have also been based on the pooling of unadjusted estimates and thus susceptible to bias including confounding by indication. As a result, it is unclear whether the observed increase in falls is causally related to such drug use versus the underlying conditions or patients for which the drugs are treating.

With the aim of evaluating its effectiveness as a single falls prevention strategy, we conducted this systematic review to answer the following: 'In older adults aged 65 years or older, does deprescribing and the withdrawal of FRIDs decrease the risk of falls compared with usual care and continuation of these drugs?' To the best of our knowledge, no previous systematic review has addressed this specific research question.

\section{METHODS}

This review was developed using the Cochrane Handbook and reported in accordance with the Preferred Reporting Items for Systematic Reviews and Meta-Analyses (PRISMA) guidelines. ${ }^{16} 17$ The protocol was registered in PROSPERO and previously published and described in detail. $^{18}$

\section{Search strategy}

MEDLINE, EMBASE, CINAHL and the Cochrane Central Register of Controlled Trials electronic databases were searched from inception to 1 August 2020 using a combination of Medical Subject Headings, controlled and free-text terms synonymous for the intervention. The MEDLINE search strategy is shown in online supplemental figure S1. This strategy was modified for use in other databases.

Reference lists of relevant studies, reviews and guidelines were reviewed to identify additional studies. Trial registries and geriatric medicine conference abstracts were also reviewed.

\section{Study eligibility criteria}

After pilot testing the eligibility criteria, pairs of reviewers independently conducted screening. A third reviewer resolved disagreements.

Studies were included if they were RCTs evaluating FRID deprescribing or withdrawal with the intent of reducing falls. FRID deprescribing was defined as the planned and supervised discontinuation or dose reduction of single or multiple medications thought to independently increase falls risk. $^{10-12}$

The comparator could be usual care (ie, no change in usual activities and/or no FRID withdrawal) or a control intervention not thought to reduce falls. Studies focused on adults aged $\geq 65$ years from all settings were included. Studies involving FRID withdrawal within multicomponent interventions were excluded if the effect of FRID withdrawal could not be isolated.

The primary outcomes of this review were the (1) rate of falls (defined as the total number of falls per unit of person time that falls were monitored) and (2) incidence of falls (ie, number of fallers). Secondary outcomes included the incidence of (1) fall-related fractures, (2) fall-related injuries, (3) fall-related hospitalisation, (4) adverse effects related to the withdrawal intervention (eg, disease relapse, symptomatic withdrawal).

\section{Data extraction and quality assessment}

Two reviewers independently abstracted data on study characteristics, participants, interventions, comparisons and outcomes using standardised electronic data extraction forms. Disagreements were resolved through consensus.

Two reviewed independently conducted risk of bias (RoB) assessments using the Cochrane Risk of Bias tool. ${ }^{19}$ A previously published modification to the RoB assessment was employed to estimate unclearly reported study 
methods and allow for sensitivity analysis. ${ }^{20}$ This modification involved a structured approach where a score of 'definitely low risk', 'probably low risk', 'probably high risk' or 'definitely high risk' was assigned to each RoB criterion. 'Definitely' and 'probably' scores were collapsed for both low and high RoB scores. Disagreements were resolved through consensus.

\section{Data synthesis and analysis}

The rate of falls was reported as a rate ratio (RaR) with a 95\% CI. Dichotomous outcomes (ie, incidences of falls, fall-related fracture, fall-related injury, fall-related hospitalisation and adverse effects related to the withdrawal intervention) have been reported as risk ratios (RRs) with 95\% CIs.

We used RevMan V.5.3 and the intention-to-treat principle for all statistical analyses. We conducted metaanalyses using the generic inverse variance method to allow pooling of effect estimates. A random-effects model was used given expected between-trial variations in methodological, participant and medication characteristics between studies. We had originally planned to pool data at various prespecified time intervals, but all included studies had follow-up between 6 and 12 months.

We assessed heterogeneity through visual inspection of forest plots and statistical tests. A two-tailed test with $\mathrm{p}$ value of $<0.10$ was considered significant for all $\mathrm{X}^{2}$ analyses as per recommendations from the Cochrane Handbook and the $\mathrm{I}^{2}$ was interpreted using the Cochrane Collaboration thresholds. ${ }^{16}$

Heterogeneity was explored in subgroup analyses based on five a priori hypotheses (online supplemental table S1) ${ }^{18}$ These included differences in baseline propensity for falls as influenced by (1) a history of recurrent falls (eg, known faller or not) or (2) place of residence or care (eg, community, long-term care); differences in the intervention as influenced by (3) specific medication class(es) chosen for withdrawal and (4) preceding medication review by a clinician for FRID withdrawal appropriateness; as well as differences in methodology based on (5) definitions used for 'falls' (eg, observed vs self-reported). We assessed the credibility of any apparent subgroup effects using 11 previously published criteria recommended by the Cochrane Handbook. ${ }^{21}$

A priori sensitivity analyses were conducted to explore the impact of low versus high RoB based on blinding and attrition. Studies did not report per-protocol results that would allow for our planned intention-to-treat versus perprotocol sensitivity analysis. The impact of using a fixedeffects versus random-effects model was explored in a post hoc sensitivity analysis.

The confidence in effect estimates for each reported outcome was assessed using the Grading of Recommendations, Assessment, Development and Evaluation (GRADE) approach. ${ }^{22}$

\section{Patient and public involvement}

Patients and the public were not involved in this review.

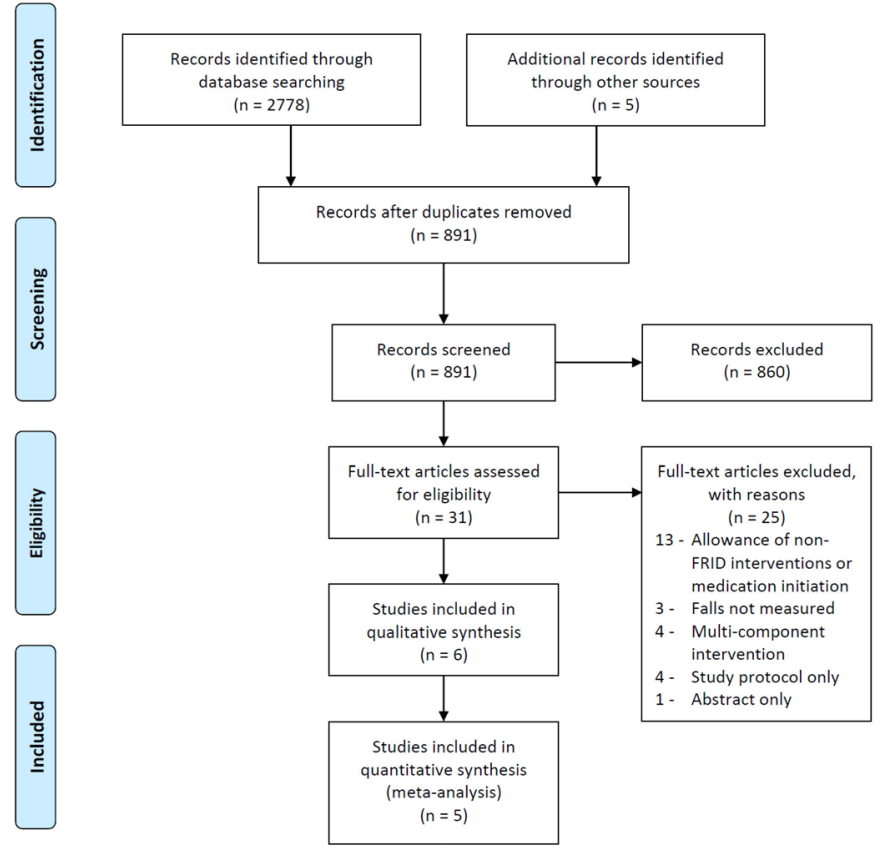

Figure 1 PRISMA flow diagram of study selection process. FRID, fall-risk increasing drug; PRISMA, Preferred Reporting Items for Systematic Reviews and Meta-Analyses.

\section{RESULTS}

Of 891 citations identified, 31 were relevant for full text review and 6 met eligibility criteria $(\kappa=0.79$, 95\% CI 0.51 to 1.00 , substantial agreement). One study was available as an abstract, but it did not report its falls data. ${ }^{23}$ Data were requested from the authors, but we did not receive a response. The PRISMA flow diagram summarising our search results is shown in figure 1.

\section{Study characteristics}

The included trials in our review are described in table 1.

Three studies were individually randomised, while two studies were cluster randomised by either nursing home or health centre. Studies ranged in size from 80 to 612 participants. With exception of one study, ${ }^{24}$ studies were multicentre involving 144 sites and 4 countries. All were conducted in the community setting except for one conducted in long-term care. ${ }^{25}$ Follow-up periods ranged from 6 to 12 months.

Overall, there were 1305 participants across all trials. Most were women (>70\%) and had a falls history (78.9\%). Several key confounders were not reported in the studies including: (1) baseline number and types of FRIDs, (2) baseline number of medications, and (3) baseline number and types of comorbidities. All these factors are thought to potentially modify falls risk. ${ }^{26} 27$

All interventions included a preceding assessment for FRID deprescribing appropriateness. This was conducted by physicians in two trials and pharmacists in three trials. Three trials tried to withdraw any FRID, while others focused on sedative-hypnotics, antipsychotics or antidepressants. Successful discontinuation and adherence to deprescribing protocols were low in all studies. Rates of 


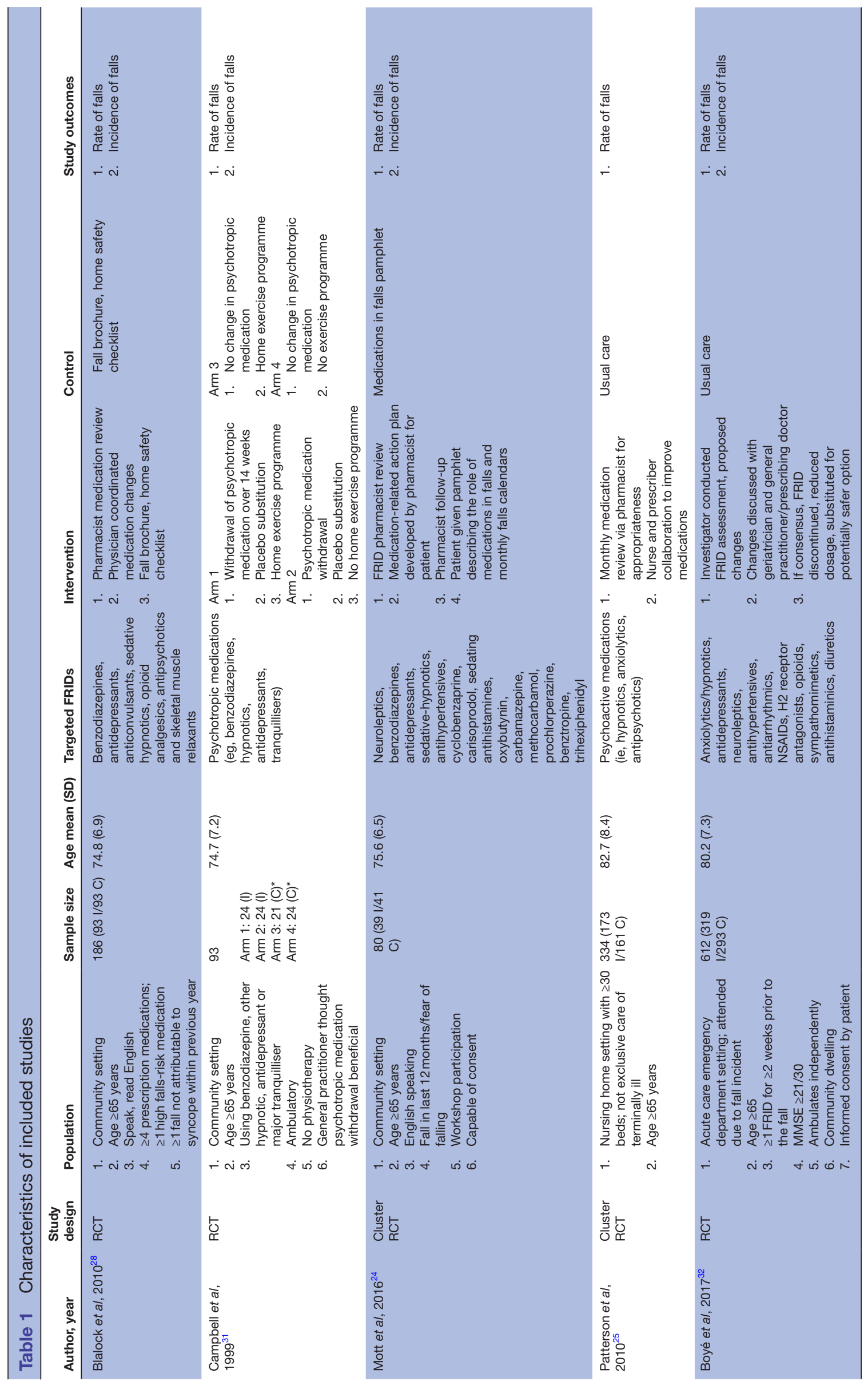


1.1 Falls Rate

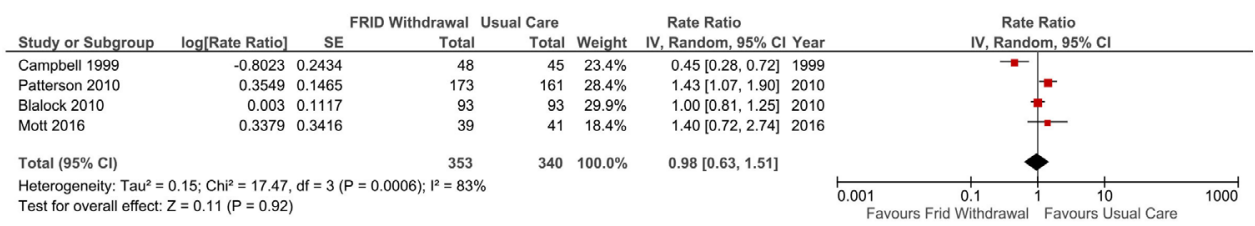

2.1 Falls Incidence - Risk Ratio

\begin{tabular}{|c|c|c|c|c|c|c|c|c|}
\hline \multirow[b]{2}{*}{ Study or Subgroup } & \multicolumn{2}{|c|}{ FRID Withdrawal } & \multicolumn{2}{|c|}{ Usual Care } & \multirow[b]{2}{*}{ Weight } & \multirow{2}{*}{$\begin{array}{c}\text { Risk Ratio } \\
\text { M-H, Random, } 95 \% \mathrm{CI}\end{array}$} & \multirow{2}{*}{\multicolumn{2}{|c|}{$\begin{array}{c}\text { Risk Ratio } \\
\text { M-H, Random, } 95 \% \mathrm{Cl}\end{array}$}} \\
\hline & Events & Total & Events & Total & & & & \\
\hline Campbell 1999 & 11 & 48 & 17 & 45 & $8.4 \%$ & $0.61[0.32,1.15]$ & & \\
\hline Blalock 2010 & 53 & 93 & 52 & 93 & $39.3 \%$ & $1.02[0.79,1.31]$ & & \\
\hline Mott 2016 & 11 & 39 & 10 & 41 & $6.4 \%$ & $1.16[0.55,2.41]$ & & \\
\hline Boyé 2017 & 115 & 319 & 91 & 293 & $45.9 \%$ & $1.16[0.93,1.45]$ & & \\
\hline Total $(95 \% \mathrm{Cl})$ & & 499 & & 472 & $100.0 \%$ & $1.04[0.86,1.26]$ & & \\
\hline Total events & 190 & & 170 & & & & & \\
\hline $\begin{array}{l}\text { Heterogeneity: Tau } \\
\text { Test for overall effec }\end{array}$ & $\begin{array}{l}0.01 ; \mathrm{Chi}^{2}= \\
\mathrm{Z}=0.44(\mathrm{P}\end{array}$ & $\begin{array}{l}70, \mathrm{df}= \\
66 \text { ) }\end{array}$ & $3(P=0$. & & & & 0.1 & 100 \\
\hline
\end{tabular}

2.2 Falls Incidence - Risk Difference

\begin{tabular}{|c|c|c|c|c|c|c|c|c|}
\hline \multirow[b]{2}{*}{ Study or Subgroup } & \multicolumn{2}{|c|}{ FRID Withdrawal } & \multicolumn{2}{|c|}{ Usual Care } & \multirow[b]{2}{*}{ Weight } & \multirow{2}{*}{$\begin{array}{l}\text { Risk Difference } \\
\text { M-H, Random, } 95 \% \mathrm{Cl}\end{array}$} & \multirow{2}{*}{$\begin{array}{c}\text { Risk Difference } \\
\text { M-H, Random, } 95 \% \mathrm{Cl}\end{array}$} & \\
\hline & Events & Total & Events & Total & & & & \\
\hline Campbell 1999 & 11 & 48 & 17 & 45 & $14.2 \%$ & $-0.15[-0.33,0.04]$ & & \\
\hline Blalock 2010 & 53 & 93 & 52 & 93 & $21.8 \%$ & $0.01[-0.13,0.15]$ & & \\
\hline Mott 2016 & 11 & 39 & 10 & 41 & $13.2 \%$ & $0.04[-0.15,0.23]$ & & \\
\hline Boyé 2017 & 115 & 319 & 91 & 293 & $50.9 \%$ & $0.05[-0.02,0.12]$ & & \\
\hline Total $(95 \% \mathrm{Cl})$ & & 499 & & 472 & $100.0 \%$ & $0.01[-0.06,0.09]$ & & \\
\hline Total events & 190 & & 170 & & & & & \\
\hline $\begin{array}{l}\text { Heterogeneity: } \text { Tau }^{2} \\
\text { Test for overall effect }\end{array}$ & $\begin{array}{l}0.00 ; \mathrm{Chi}^{2}= \\
\mathrm{z}=0.31(\mathrm{P}=\end{array}$ & $\begin{array}{l}86, \mathrm{df}= \\
7.76)\end{array}$ & & & & & -0.5 & 0.5 \\
\hline
\end{tabular}

3.1 Fall-Related Injuries

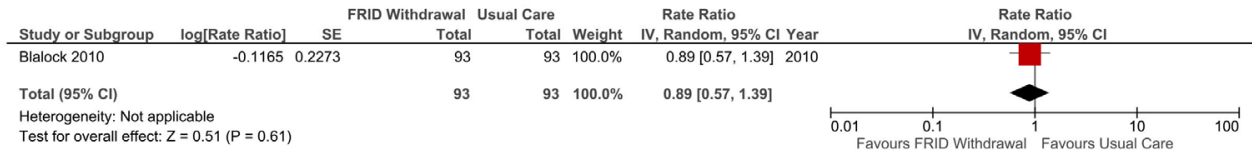

Figure 2 Forest plots of FRID withdrawal versus usual care. FRID, fall-risk increasing drug.

complete discontinuation of at least one FRID ranged from $10 \%$ to $40 \%$.

In terms of our study outcomes, four trials measured the rate of falls and four measured falls incidence. One trial reported fall-related injuries. ${ }^{28}$ Fall-related fractures, fall-related hospitalisation or deprescribing-related adverse effects were not measured by any of the trials.

\section{Summary of findings}

Rate and incidence of falls

Four studies reported the effect of deprescribing FRIDs on the rate of falls. Deprescribing FRIDs did not reduce the rate of falling ( $\mathrm{RaR} 0.98,95 \%$ CI 0.63 to 1.51 ; figure 2analysis 1.1$)$. Considerable statistical heterogeneity was present $\left(\chi^{2}=17.47, \mathrm{p}=0.0006, \mathrm{I}^{2}=83 \%\right)$ and subsequently explored in subgroup analysis.

Four studies reported the effect of deprescribing FRIDs on the risk of falls as measured by falls incidence. Deprescribing FRIDs did not reduce the incidence of falls (RR 1.04, 95\% CI 0.86 to $1.26, \mathrm{I}^{2}=19 \%, \chi^{2}=3.70$, $\mathrm{p}=0.30$; figure 2-analysis 2.1). In absolute terms, there was a non-significant risk difference increase of 0.01 ( $95 \%$ CI -0.06 to $0.09, \mathrm{I}^{2}=22 \%, \mathrm{p}=0.76$; figure 2 -analysis 2.2).
Rate of injurious falls

One trial reported the effect of deprescribing FRIDs on fall-related injuries. ${ }^{28}$ Deprescribing FRIDs did not reduce the rate of fall-related injuries (RaR $0.89,95 \%$ CI 0.57 to 1.39 ; figure 2-analysis 3.1 ). This trial did not report data that would allow for any of our preplanned subgroup analyses.

\section{RoB assessment}

Figure 3 summarises our RoB assessments. All studies were deemed at high RoB in at least one domain. The overall mean weighted kappa across all assessments was 0.67 (moderate agreement). For individual RoB assessments, kappa ranged from 0 to 0.85 . Inter-rater agreement is actually higher than indicated by the calculated scores due to the 'kappa coefficient paradox'. ${ }^{29}{ }^{30}$ Low kappas (eg, $\kappa=0$ ) occurred despite high levels of observed agreement (eg, $\geq 80 \%$ agreement) for two RoB assessments. True agreement is falsely attributed to chance agreement by the kappa calculation when there is substantial imbalance in marginal ratings.

For falls rate and incidence, all studies except one $\mathrm{e}^{31}$ were judged at high RoB for lack of blinding of participants, personnel and outcome assessors. It is unclear 

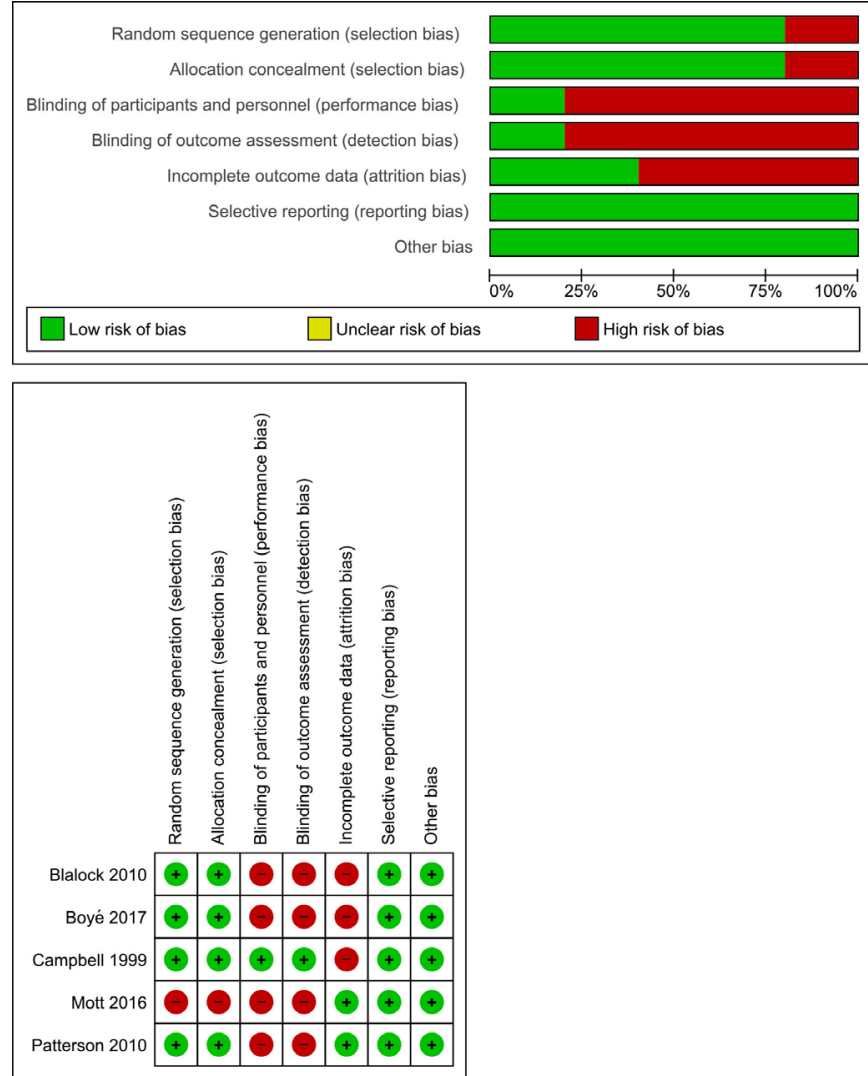

Figure 3 Risk of bias assessments.

whether blinding could have impacted behaviour or perceptions (eg, activity risk-level, nocebo effect). Risk of ascertainment bias was high in one study ${ }^{25}$ (ie, no standardised falls definition was used), but all other studies used methods accepted to be low RoB (ie, falls recorded daily on postcards or calendars). Risk of attrition bias was deemed high in three studies based on high or unbalanced lost to follow-up rates. ${ }^{28} 3132$

\section{Publication bias}

Since less than 10 eligible studies were found, a funnel plot was not constructed due to an inability to make meaningful conclusions about publication bias.

\section{Subgroup analyses and exploration of heterogeneity}

Our prespecified subgroup analyses did not adequately explain the statistical heterogeneity observed results for the rate and incidence of falls (online supplemental figure S2). Deprescribing FRIDs appeared more effective when a preceding medication review was conducted by physicians compared with pharmacists $(\mathrm{p}=0.0004$, $\mathrm{I}^{2}=91.9 \%$, analysis 1.5 ), while psychotropic withdrawal appeared more effective than strategies withdrawing any FRID ( $\mathrm{p}=0.08, \mathrm{I}^{2}=67.8 \%$, analysis 2.3 ). However, in both analyses, only 6 of 11 subgroup credibility criteria were met and each subgroup was limited to one trial with less than 100 participants (online supplemental table S2). We, therefore, judged the credibility that these subgroup effects are real as poor and uncertain.
The available data did not permit subgroup analyses by place of residence or falls ascertainment method. The other subgroup analyses showed no evidence of difference beyond that due to chance.

\section{Sensitivity analyses}

Our sensitivity analyses are shown in online supplemental figure S3. The incorporation of trials with high risk of performance bias appeared to mask the potential benefit of deprescribing FRIDs on reducing the incidence and rate of falls, while the trials with high risk of attrition bias appeared to mask a potential increase in falls rate with deprescribing FRIDs. These results should be interpreted cautiously and definitive conclusions cannot be made. Data from trials with low risk of performance bias were limited to one trial with less than 100 participants, and data from trials with low risk of attrition bias were limited to two trials with less than 450 participants overall.

A post-hoc sensitivity analysis examining the impact of using a fixed-effects versus random-effects model did not change conclusions regarding the effect of deprescribing FRIDs on the rate or incidence of falls.

\section{Quality of evidence}

The GRADE evidence profile is shown in table 2.

We judged the quality of evidence to be low or very low for all outcomes (falls rates, falls incidence and fallrelated injuries) after rating down for RoB, inconsistency and imprecision.

We believe the optimal information size (OIS) to make definitive conclusions on the effect of deprescribing FRIDs has not yet been met as the body of evidence is based on fewer than 2000 participants and less than 400 events. ${ }^{33}$ This is based on the OIS calculation figure recommended by the GRADE guidelines using a wellestablished control falls event rate of $30 \%$ described in the literature and conservative RR reduction of $20 \%$ (assuming $\alpha=0.05$ and $\beta=0.2$ ). ${ }^{34} 35$

\section{DISCUSSION}

This systematic review sought to determine whether deprescribing FRIDs decreased the risk of falls in older adults and found that there is a lack of robust high-quality evidence to support or refute the deprescribing of FRIDs alone as an effective falls prevention strategy. Incorporating data from 5 RCTs involving 1305 participants aged $\geq 65$ years, our meta-analyses indicate that a FRID deprescribing strategy did not significantly change the rate of falls (RaR $0.98,95 \%$ CI 0.63 to 1.51 ) nor the risk of falling (risk difference (RD) $0.01,95 \%$ CI -0.06 to 0.09 ) over a follow-up period of 6-12 months. Although this intervention focuses on those medications thought to be associated with falls, the uncertainty of its effect on falls and conclusions of current lack of evidence of effectiveness are similar to previous systematic reviews evaluating the effectiveness of medication reviews that had a broader 


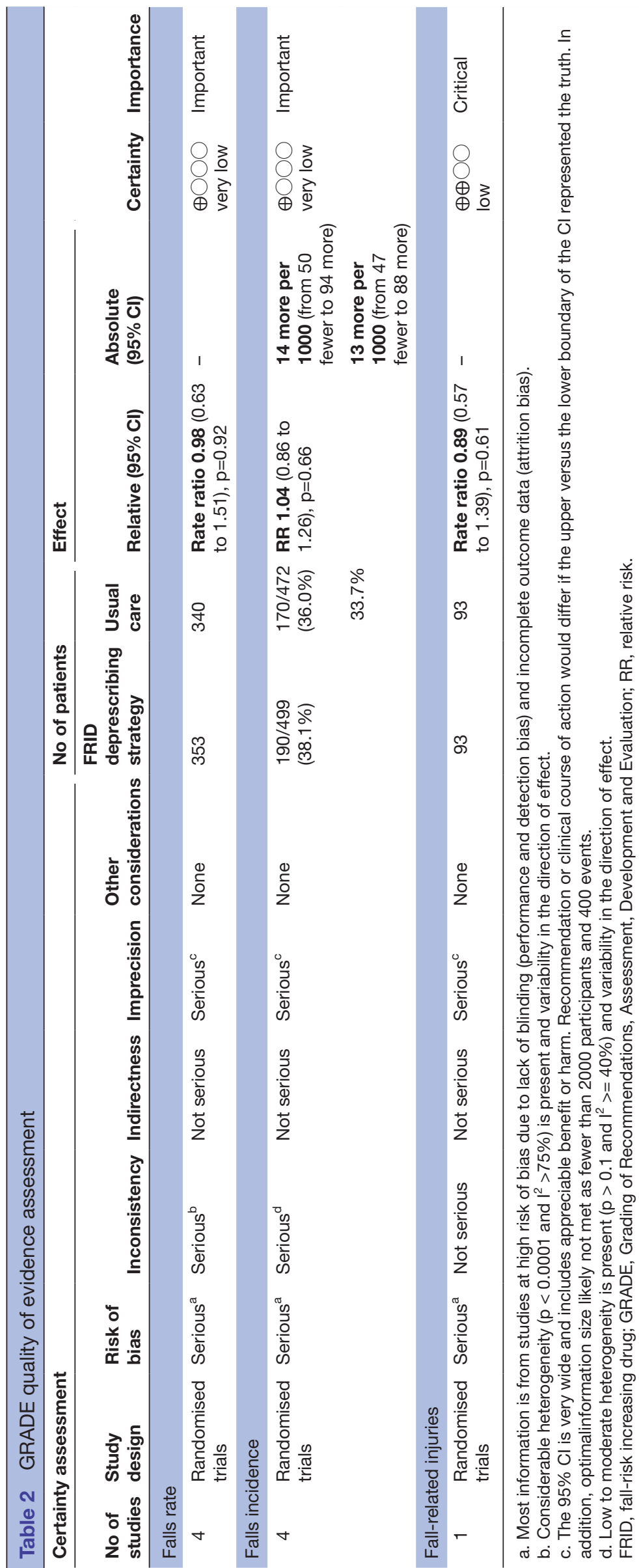


focus on reducing polypharmacy and potentially inappropriate prescribing (ie, not focused solely on FRIDs). ${ }^{936}$

There is also a significant absence of evidence for clinically and patient-important outcomes such as fall-related injuries, fractures and hospitalisations. The only trial to date that evaluated the rate of fall-related injuries did not demonstrate a statistically significant effect (RaR $0.89,95 \%$ CI 0.57 to 1.39$).^{28}$ Our search found no trials measuring the impact on fall-related fractures, fall-related hospitalisations or adverse effects related to a FRID deprescribing strategy. Although this may be rooted in the difficulty of conducting RCTs powered for such outcomes, their measurement and reporting are still important to inform systematic review meta-analyses that could lead to more precise estimates.

Based on low-quality evidence, it is unclear whether deprescribing FRIDs as a single intervention leads to any appreciable clinically important benefit or harm. Our current best effect estimates for falls rate and incidence are centred around no appreciable difference (ie, $R a R \approx 1, R R \approx 1, R D \approx 0$ ). Although seemingly logical to assume, reducing isolated risk factors may not necessarily lead to a reduction in falls and fall-related complications. The absence of change in the incidence of hip fractures after state-wide regulatory action on benzodiazepine prescribing in the USA that reduced benzodiazepine use by $60.3 \%$ is a real-world example of this phenomenon and the complexity of exposure-outcome relationships. ${ }^{37}$

Our findings likely reflect the multifactorial nature of falls and the varying risk of different FRIDs. It is unclear as to what degree a particular risk factor or combination of risk factors (eg, specific FRIDs) must be reduced to produce an appreciable change in falls. Medications may only have conditional or contributory causality to falls. It may be that medication-related interventions work best in combination with other interventions or only in specific contexts.

Only one trial ${ }^{31}$ included in our review demonstrated a statistically significant benefit with deprescribing FRIDs. This was also the only trial to use study capsules to operationalise blinded deprescribing of FRIDs in participants, research personnel and outcome assessors. Its results might be more reflective of the true potential physiological effect of deprescribing FRIDs because it minimised the risk of performance bias. However, the magnitude of benefit achievable in the non-research setting at this time may be closer to those seen in the unblinded trials due to the strong psychological and behavioural factors (eg, nocebo effect) that may hinder successful deprescribing. Further advances in implementation science and behavioural change strategies are likely needed to facilitate medication optimisation.

These results raise several questions about the presumed effectiveness of deprescribing FRIDs as an isolated falls prevention strategy. Given the amount of resources being invested into falls prevention initiatives around the world, clinicians and organisations should examine: (1) what is the strength of evidence supporting their current activities, (2) whether these activities are cost-effective and (3) whether resources are being appropriately prioritised to those interventions shown to provide the most value. This should also be applied to what is being required of healthcare organisations in national accreditation standards (eg, Joint Commission, Accreditation Canada) to help direct and encourage optimal use of limited healthcare resources.

Clinicians and policymakers need to consider the current lack of strong evidence for deprescribing FRIDs as an isolated intervention for the specific purpose of reducing falls, particularly in patients who may be very reluctant or who have strong indications for specific FRIDs. FRID reduction is one out of many possible interventions that need to be considered. As with prescribing medications, deprescribing is a skill and comes with the potential for harm as well as benefit. ${ }^{38}$ Thoughtful consideration of the goals, appropriateness and safety of deprescribing is important. ${ }^{39}$ Our results highlight the need for a comprehensive and individualised approach to falls. Multicomponent interventions are ideal, but interventions may need to be prioritised depending on time, resources and context.

Despite insufficient evidence to support or refute the deprescribing of FRIDs for falls prevention, our results do not mean that clinicians should avoid deprescribing FRIDs. There may be many other reasons to deprescribe these medications. These include avoidance of adverse drug events, improvements in cognition, increased medication adherence and drug costs savings. It is also unclear whether medication review and management with a broader focus on reducing polypharmacy and potentially inappropriate prescribing in older adults may be beneficial in preventing falls. Some RCTs with such interventions have shown a reduction of falls risk, while others have not demonstrated a significant difference. ${ }^{40-46}$

Our review highlights the need for future FRID deprescribing trials that evaluate patient-important outcomes (eg, injuries, fractures and hospitalisations). Greater attention to optimal design and reporting is needed to minimise RoB and enhance our interpretation of the results. Examples include improved reporting of confounding baseline characteristics and intervention fidelity (eg, number and types of FRIDs, degree and duration of dose reduction). Deprescribing is challenging and extra measures are likely needed to improve successful intervention adherence and follow-up.

\section{Strengths and limitations}

Our review has limitations. There was variation in the operationalisation of FRID deprescribing and degree of success achieved (eg, dose reduction only, completion discontinuation, non-adherence). This presumably makes the detection of any potential benefit less likely and our conclusions more conservative. However, the effect estimates are likely more indicative of what might be expected outside of the research setting. These phenomena likely represent the real-life challenges of 
deprescribing (especially with certain types of FRIDs such as psychotropics or opioids). Moreover, our ability to assess for confounders modifying falls risk was limited due to inconsistent reporting of relevant baseline characteristics and lack of patient-level data. Lastly, our ability to make definitive conclusions is limited because the total sample size across studies for each outcome did not yet meet our calculated estimate for the required optimal information size.

Our review has several strengths. First, our search was comprehensive and we included a rigorous grey literature search for unpublished studies. Second, we employed optimal analytical and interpretational approaches including duplicate assessment, subgroup credibility criteria and optimal information size considerations. Third, unlike previous medication-focused reviews, we applied the GRADE approach to assess the quality of evidence and our degree of confidence in the results.

\section{CONCLUSIONS}

Our systematic review found that deprescribing FRIDs as an isolated strategy results in little to no difference in the rate and risk of falls or fall-related injuries, but the evidence is still sparse and very low quality. Additional well-designed studies are needed to reach the optimal information size to reduce uncertainty about this intervention and establish its relative importance in the range of possible interventions that can be employed by clinicians and health systems to reduce falls.

\section{Author affiliations}

${ }^{1}$ Division of Geriatric Medicine, Department of Medicine, McMaster University, Hamilton, Ontario, Canada

${ }^{2}$ Department of Health Research Methods, Evidence and Impact, McMaster University, Hamilton, Ontario, Canada

${ }^{3}$ GERAS Centre for Aging Research, Hamilton Health Sciences, Hamilton, Ontario, Canada

${ }^{4}$ School of Rehabilitation Sciences, McMaster University Faculty of Health Sciences, Hamilton, Ontario, Canada

${ }^{5}$ Department of Medicine, Queen's University, Kingston, Ontario, Canada

${ }^{6}$ Division of Geriatric Medicine, Department of Medicine, University of Toronto, Toronto, Ontario, Canada

${ }^{7}$ Division of Clinical Pharmacology and Toxicology, Department of Medicine, McMaster University, Hamilton, Ontario, Canada

Twitter Justin Lee @JustinLeeMD, Ahmed Negm @_negm_and Eric K C Wong @ EricWong_MD

Acknowledgements The authors would like to thank Lois Cottrell, medical librarian at St Joseph's Healthcare Hamilton, Charlton Campus, Hamilton, Ontario, Canada; and Andrea McLellan, Head of Collection and Technical Services, School of Medicine liaison, at McMaster University, Hamilton, Ontario, Canada, for their assistance with the development of the search strategy.

Contributors $\mathrm{JL}$ conceptualised the study. $\mathrm{JL}$ and $\mathrm{AH}$ designed and developed the protocol. RP and EKCW assisted with citation review. RP and AMN assisted with data extraction, risk of bias assessment and certainty of evidence grading. All authors contributed to the analysis and interpretation of results. JL drafted the initial manuscript and all authors contributed to its revision and final approval.

Funding The authors have not declared a specific grant for this research from any funding agency in the public, commercial or not-for-profit sectors.

Competing interests None declared.

Patient consent for publication Not required.
Provenance and peer review Not commissioned; externally peer reviewed.

Data availability statement Data sharing not applicable as no datasets generated and/or analysed for this study.

Supplemental material This content has been supplied by the author(s). It has not been vetted by BMJ Publishing Group Limited (BMJ) and may not have been peer-reviewed. Any opinions or recommendations discussed are solely those of the author(s) and are not endorsed by BMJ. BMJ disclaims all liability and responsibility arising from any reliance placed on the content. Where the content includes any translated material, BMJ does not warrant the accuracy and reliability of the translations (including but not limited to local regulations, clinical guidelines, terminology, drug names and drug dosages), and is not responsible for any error and/or omissions arising from translation and adaptation or otherwise.

Open access This is an open access article distributed in accordance with the Creative Commons Attribution Non Commercial (CC BY-NC 4.0) license, which permits others to distribute, remix, adapt, build upon this work non-commercially, and license their derivative works on different terms, provided the original work is properly cited, appropriate credit is given, any changes made indicated, and the use is non-commercial. See: http://creativecommons.org/licenses/by-nc/4.0/.

\section{ORCID iDs}

Justin Lee http://orcid.org/0000-0002-8488-6018

Anne Holbrook http://orcid.org/0000-0002-3371-4187

\section{REFERENCES}

1 Hill KD, Wee R. Psychotropic drug-induced falls in older people: a review of interventions aimed at reducing the problem. Drugs Aging 2012;29:15-30.

2 Florence CS, Bergen G, Atherly A, et al. Medical costs of fatal and nonfatal falls in older adults. J Am Geriatr Soc 2018;66:693-8.

3 National Institute for Health and Care Excellence. Falls in older people: assessing risk and prevention, 2013.

4 Public Health Agency of Canada. Seniors' Falls in Canada, 2014.

5 Panel on Prevention of Falls in Older Persons, American Geriatrics Society and British Geriatrics Society. Summary of the updated American geriatrics Society/British geriatrics Society clinical practice guideline for prevention of falls in older persons. J Am Geriatr Soc 2011;59:148-57.

6 Guirguis-Blake JM, Michael YL, Perdue LA. Interventions to prevent falls in older adults. JAMA 1705;2018:319.

7 Campbell AJ, Robertson MC. Fall prevention: single or multiple interventions? single interventions for fall prevention. J Am Geriatr Soc 2013;61:281-4.

8 Tricco AC, Thomas SM, Veroniki AA, et al. Comparisons of interventions for preventing falls in older adults. JAMA 2017;318:1687.

9 Gillespie LD, Robertson MC, Gillespie WJ, et al. Interventions for preventing falls in older people living in the community. Cochrane Database Syst Rev 2012;9:CD007146.

10 Leipzig RM, Cumming RG, Tinetti ME. Drugs and falls in older people: a systematic review and meta-analysis: I. psychotropic drugs. J Am Geriatr Soc 1999;47:30-9.

11 Leipzig RM, Cumming RG, Tinetti ME. Drugs and falls in older people: a systematic review and meta-analysis: II. cardiac and analgesic drugs. J Am Geriatr Soc 1999;47:40-50.

12 Woolcott JC, Richardson KJ, Wiens MO, et al. Meta-analysis of the impact of 9 medication classes on falls in elderly persons. Arch Intern Med 2009;169:1952-60.

13 de Vries M, Seppala LJ, Daams JG, et al. Fall-Risk-Increasing drugs: a systematic review and meta-analysis: I. cardiovascular drugs. J Am Med Dir Assoc 2018;19:371.e1-371.e9.

14 Seppala LJ, Wermelink AMAT, de Vries M, et al. Fall-Risk-Increasing drugs: a systematic review and meta-analysis: II. psychotropics. J Am Med Dir Assoc 2018;19:371.e11-17.

15 Seppala LJ, van de Glind EMM, Daams JG, et al. Fall-risk-increasing drugs: a systematic review and meta-analysis: III. others. J Am Med Dir Assoc 2018;19:372.e1-8.

16 The Cochrane Collaboration. Cochrane handbook for systematic reviews of interventions version 5.1.0 [updated March 2011], 2011.

17 Moher D, Liberati A, Tetzlaff J, et al. Preferred reporting items for systematic reviews and meta-analyses: the PRISMA statement. Ann Intern Med 2009;151:264-9.

18 Lee JY, Holbrook A. The efficacy of fall-risk-increasing drug (FRID) withdrawal for the prevention of falls and fall-related complications: protocol for a systematic review and meta-analysis. Syst Rev 2017;6:33. 
19 Higgins JPT, Altman DG, Gøtzsche PC, et al. The Cochrane Collaboration's tool for assessing risk of bias in randomised trials. BMJ 2011;343:d5928.

20 Akl EA, Sun X, Busse JW, et al. Specific instructions for estimating unclearly reported blinding status in randomized trials were reliable and valid. J Clin Epidemiol 2012;65:262-7.

21 Sun X, Briel M, Walter SD, et al. Is a subgroup effect believable? Updating criteria to evaluate the credibility of subgroup analyses. BMJ 2010;340:c117.

22 Guyatt G, Oxman AD, Akl EA, et al. GRADE guidelines: 1. Introduction-GRADE evidence profiles and summary of findings tables. J Clin Epidemiol 2011;64:383-94.

23 Streim JE, Filippo S, Have T. Antidepressant discontinuation associated with cognitive decline in older adult residents of longterm care facilities. Am J Geriatr psychiatry 2012;20:S147-8.

24 Mott DA, Martin B, Breslow R, et al. Impact of a medication therapy management intervention targeting medications associated with falling: results of a pilot study. J Am Pharm Assoc 2016;56:22-8.

25 Patterson SM, Hughes CM, Crealey G, et al. An evaluation of an adapted U.S. model of pharmaceutical care to improve psychoactive prescribing for nursing home residents in Northern Ireland (fleetwood Northern Ireland study). J Am Geriatr Soc 2010;58:44-53.

26 Tinetti ME, Kumar C. The patient who falls. JAMA 2010;303:258-4.

27 Chen Y, Zhu L-L, Zhou Q. Effects of drug pharmacokinetic/ pharmacodynamic properties, characteristics of medication use, and relevant pharmacological interventions on fall risk in elderly patients. Ther Clin Risk Manag 2014;10:437-48.

28 Blalock SJ, Casteel C, Roth MT, et al. Impact of enhanced pharmacologic care on the prevention of falls: a randomized controlled trial. Am J Geriatr Pharmacother 2010;8:428-40.

29 Feinstein AR, Cicchetti DV. High agreement but low kappa: I. The problems of two paradoxes. J Clin Epidemiol 1990;43:543-9.

30 Tang W, Hu J, Zhang H, et al. Kappa coefficient: a popular measure of rater agreement. Shanghai Arch Psychiatry 2015;27:62-7.

31 Campbell AJ, Robertson MC, Gardner MM, et al. Psychotropic medication withdrawal and a home-based exercise program to prevent falls: a randomized, controlled trial. J Am Geriatr Soc 1999;47:850-3.

32 Boyé NDA, van der Velde N, de Vries OJ, et al. Effectiveness of medication withdrawal in older fallers: results from the improving medication prescribing to reduce risk of falls (IMPROveFALL) trial. Age Ageing 2017;46:142-6.

33 loannidis J, Lau J. Evolution of treatment effects over time: empirical insight from recursive cumulative metaanalyses. Proc Natl Acad Sci U S A 2001;98:831-6.
34 Guyatt GH, Oxman AD, Kunz R, et al. GRADE guidelines 6. Rating the quality of evidence-imprecision. J Clin Epidemiol 2011;64:1283-93.

35 O'Loughlin JL, Robitaille Y, Boivin JF, et al. Incidence of and risk factors for falls and injurious falls among the community-dwelling elderly. Am J Epidemiol 1993;137:342-54.

36 Guirguis-Blake JM, Michael YL, Perdue LA. Interventions to prevent falls in community-dwelling older adults: a systematic review for the US. preventive services task force. Agency Health Res Qual 2018:W6.

37 Wagner AK, Ross-Degnan D, Gurwitz JH, et al. Effect of New York State regulatory action on benzodiazepine prescribing and hip fracture rates. Ann Intern Med 2007;146:96-103.

38 Reeve E, Moriarty F, Nahas R, et al. A narrative review of the safety concerns of deprescribing in older adults and strategies to mitigate potential harms. Expert Opin Drug Saf 2018;17:39-49.

39 Lee JY, Farrell B, Holbrook AM. De-prescribing benzodiazepine receptor agonists taken for insomnia: a review and key messages from practice guidelines. Polish Arch Intern Med 2018;129:839-45.

40 Pit SW, Byles JE, Henry DA, et al. A quality use of medicines program for general practitioners and older people: a cluster randomised controlled trial. Med J Aust 2007;187:23-30.

41 Weber V, White A, Mcllvried R. An electronic medical record (EMR)-based intervention to reduce polypharmacy and falls in an ambulatory rural elderly population. J Gen Intern Med 2008;23:399-404.

42 Zermansky AG, Alldred DP, Petty DR, et al. Clinical medication review by a pharmacist of elderly people living in care homes--randomised controlled trial. Age Ageing 2006;35:586-91.

43 Frankenthal D, Lerman Y, Kalendaryev E, et al. Intervention with the screening tool of older persons potentially inappropriate prescriptions/screening tool to alert doctors to right treatment criteria in elderly residents of a chronic geriatric facility: a randomized clinical trial. J Am Geriatr Soc 2014:62:1658-65.

44 Michalek C, Wehling M, Schlitzer J, et al. Effects of 'Fit fOR The Aged' (FORTA) on pharmacotherapy and clinical endpoints-a pilot randomized controlled study. Eur J Clin Pharmacol 2014;70:1261-7.

45 Meredith S, Feldman P, Frey D, et al. Improving medication use in newly admitted home healthcare patients: a randomized controlled trial. J Am Geriatr Soc 2002;50:1484-91.

46 Sjöberg C, Wallerstedt SM. Effects of medication reviews performed by a physician on treatment with fracture-preventing and fall-riskincreasing drugs in older adults with hip fracture-a randomized controlled study. J Am Geriatr Soc 2013;61:1464-72. 